\title{
Clinical Efficacies of the Minimal Retroperitoneal Approach for Infectious Spondylodiscitis: A Clinical Case Series
}

\author{
Tsunehiko Konomi $^{1)}$, Shinjiro Kaneko ${ }^{12)}$, Amir Fariz Zakaria ${ }^{1223)}$, Kanehiro Fujiyoshi ${ }^{1)}$, Junichi Yamane ${ }^{1)}$, \\ Takashi Asazuma ${ }^{1)}$ and Yoshiyuki Yato ${ }^{1)}$ \\ 1) Department of Orthopaedic Surgery, Murayama Medical Center, National Hospital Organization, Tokyo, Japan \\ 2) Department of Spine and Spinal Cord Surgery, Fujita Health University Hospital, Toyoake, Japan \\ 3) Department of Orthopaedic Surgery, Hospital Raja Permaisuri Bainun, Ipoh, Malaysia
}

\begin{abstract}
:
Introduction: An anterior surgical approach for severe infectious spondylodiscitis in the lumbar region is optimal but not always atraumatic. The aim of this study was to evaluate the efficacy and safety of a minimal anterior-lateral retroperitoneal approach, also known as a surgical approach for oblique lumbar interbody fusion, for cases with severe infectious spondylodiscitis with osseous defects.

Methods: Twenty-four consecutive patients who underwent anterior debridement and spinal fusion with an autologous strut bone graft for infectious spondylodiscitis with osseous defects were reviewed retrospectively. Eleven patients underwent the minimal retroperitoneal approach (Group M), and 13 underwent the conventional open approach (Group C). Periand postoperative clinical outcomes, that is, estimated blood loss (EBL), operative time (OT), creatine kinase (CK) level, visual analog scale (VAS), and rates of bone union and additional posterior instrumentation, were evaluated, and the differences between both groups were assessed statistically.

Results: Mean EBL, serum CK on the $1^{\text {st }}$ postoperative day, and VAS on the $14^{\text {th }}$ postoperative day were $202.1 \mathrm{~mL}, 390.9$ IU/L, and $9.5 \mathrm{~mm}$ in Group M and $648.3 \mathrm{~mL}, 925.5 \mathrm{IU} / \mathrm{L}$, and $22.3 \mathrm{~mm}$ in Group C, respectively, with statistically significant differences between the groups. There were no statistically significant intergroup differences in OT and rates of bone union and additional posterior instrumentation.

Conclusions: Anterior debridement and spinal fusion using the minimal retroperitoneal approach is a useful and safe surgical technique. Although a preponderance of the minimal approach regarding early bone union is not validated, this technique has the advantages of conventional open surgery, but reduces blood loss, muscle injury, and pain postoperatively.
\end{abstract}

Keywords:

anterior spinal fusion, infectious spondylodiscitis, oblique lumbar interbody fusion

Spine Surg Relat Res 2021; 5(3): 176-181 dx.doi.org/10.22603/ssrr.2020-0134

\section{Introduction}

The incidence of infectious spondylitis is increasing among elderly individuals because of population aging and an increase in the number of patients with multiple comorbidities $^{1-3)}$. The most frequent focus of this lesion is the vertebral endplate, which then invades the intervertebral disc space owing to its vascular anatomy ${ }^{4-6)}$. Anterior lumbar surgery is an option for the management of destructive and infectious spondylodiscitis. This technique offers great exposure of the vertebral body and surrounding inter- and prever- tebral spaces ${ }^{7)}$; however, it demands highly technical skills with a large skin incision, especially when performing anterior open debridement or spinal fusion for a large osseous defect. This large skin incision is paramount to obtaining an adequate working space, which is normally deep, narrow, and with poor illumination. This approach also has the potential risk of complications such as vascular, colon, lumbar plexus, and pneumothorax injury with substantial blood loss. Furthermore, patients with multiple comorbidities undergoing major surgery often experience postsurgical complications, including relapse of infection, wound dehiscence,

Corresponding author: Tsunehiko Konomi, konomitsunehiko@gmail.com 
Table 1. Severity Grade of Spondylodiscitis Described by Pee et al. ${ }^{10}$

\begin{tabular}{cc}
\hline Grade & Definition \\
\hline I & Isolated discitis or discitis with minor destruction of the endplate \\
II & Discitis with moderate endplate destruction \\
III & Discitis with destruction of the vertebral body \\
\hline
\end{tabular}

The differentiation between "minor" and "moderate" destruction to the endplate is based on findings of the destruction of the vertebral body adjacent to the endplate noted on computed tomography scans. Destruction of the posterior or anterior wall of the vertebral body is defined as grade III.

pseudarthrosis, and instrumentation failure ${ }^{1)}$.

To avoid major complications in anterior open surgery, a minimal anterior-lateral retroperitoneal approach, which is based on the method for oblique lumbar interbody fusion (OLIF) surgery, has been attracting attention. An X-ray fluoroscopy device and a specialized retractor for OLIF enable one to access intervertebral space anterior-laterally via between the aorta and the anterior edge of the psoas muscle more directly, resulting in a reduced risk of lumbar plexus injury, vascular injury, and damage to the paraspinal musculature and posterior elements ${ }^{8,9}$. We hypothesized that this OLIF approach could be suitable even for cases with a large bone defect following infectious spondylodiscitis. In this study, we evaluated the efficacy and safety of lumbar debridement and spinal fusion surgery with an autologous strut bone graft using the minimal retroperitoneal approach for cases with severe infectious spondylodiscitis in comparison to conventional anterior open surgery.

\section{Materials and Methods}

This was a retrospective case series study of patients who underwent anterior spinal fusion surgery at a single center between April 2011 and March 2018. This study was ethically approved by the Research Ethics Committee of our institution. A total of 45 consecutive patients who underwent anterior spinal debridement and fusion with an autologous strut graft for destructive spondylodiscitis in the lumbar region following tuberculous spondylodiscitis (TS) or pyogenic spondylodiscitis (PS), with a severity grade of II or III (Table 1) according to the imaging study ${ }^{10}$, were eligible for this study. Of these 45 patients, patients treated with an anterior intervertebral cage, spacer, or any other anterior instrumentation were excluded. As a result, 24 patients were selected and divided into two groups according to the surgical procedure: 11 patients in the minimal retroperitoneal OLIF approach group (Group M), and 13 in the conventional anterior open approach group (Group C). The conventional open approach was applied until April 2014. Thereafter, the minimal retroperitoneal approach was applied. The minimum postsurgery follow-up period was 12 months. Peri- and postoperative clinical outcomes, that is, age, sex, amount of estimated blood loss (EBL), operative time (OT), creatine kinase $(\mathrm{CK})$ level at the $1^{\text {st }}$ postoperative day, visual analog scale (VAS) at the $7^{\text {th }}$ and $14^{\text {th }}$ postoperative days, rate of bone union, recurrence, and history of any additional surgery, were evaluated retrospectively from medical charts. Bone union was assessed by lateral dynamic radiography or computed tomography (CT) findings following primary surgical treatment. The criteria for radiological assessment of bone union were as follows: (1) More than $3^{\circ}$ of motion on flexion-extension in the radiograph was considered to indicate nonunion. (2) Nonunion was defined as the presence of a visible gap between the vertebral endplate and the harvest bone on a radiograph and/or a CT image ${ }^{11-13}$. Clinical outcome was evaluated using the Japanese Orthopedic Association (JOA) scores at baseline and final follow-up. The recovery rate was calculated as the following formula: (postoperative score-baseline score)/(29 [full score]-baseline score) $\times$ $100(\%)^{14)}$. The differences between both groups were assessed by Fisher's exact test for categorical variables and the unpaired $t$ test for continuous variables with the use of Prism 5.0 (GraphPad Software, Inc., La Jolla, CA, USA). A value of $P<0.05$ was considered to be statistically significant.

\section{Surgical procedure}

In Group M, patients were positioned in the lateral decubitus position. A 5-cm skin incision was made at the lateral abdominal region, at the anterior axillary line and centered on the affected disc level. This incision was parallel to the fibers of the external oblique muscle and $5 \mathrm{~cm}$ anterior to the anterior border of the vertebral body. The external oblique, internal oblique, and transverse abdominal muscles were then dissected along the direction of their fibers. The retroperitoneal space was accessed by blunt dissection, and peritoneal content was mobilized anteriorly. The psoas muscle and genitofemoral nerve were identified and mobilized posteriorly, whereas the aorta, sympathetic chain, and ureter were mobilized anteriorly. Subsequently, the intervertebral disc was exposed through this open corridor. A Kirschner wire (K-wire) was placed in the target disc space from the anterolateral corner, and its position was confirmed under fluoroscopy. Sequential dilators were placed over the K-wire and finally followed by the self-retaining retractor. The retractor was placed under illumination to ensure a bright operating field.

In Group C, patients were positioned in the lateral decubitus position on an operating table that was flexed to increase exposure between the $12^{\text {th }}$ rib and iliac crest. An oblique in- 
Table 2. Clinical Details of the Minimal Retroperitoneal Approach Group (Group M).

\begin{tabular}{ccccccc}
\hline $\begin{array}{c}\text { Case } \\
\text { No. }\end{array}$ & Age (years)/Sex & $\begin{array}{c}\text { Follow-up } \\
\text { (months) }\end{array}$ & Diagnosis & Level & Grade & $\begin{array}{c}\text { Additional Posterior } \\
\text { Instrumentation }\end{array}$ \\
\hline 1 & $84 / \mathrm{M}$ & 44 & TS & L4/5 & II & + +: Delayed Union \\
2 & $77 / \mathrm{M}$ & 36 & PS & L3/4 & II & - \\
3 & $61 / \mathrm{F}$ & 48 & PS & L2/3/4 & II & - \\
4 & $76 / \mathrm{M}$ & 48 & PS & L4/5 & II & - \\
5 & $49 / \mathrm{M}$ & 30 & TS & L3/4 & II & + +: Delayed Union \\
6 & $63 / \mathrm{F}$ & 31 & PS & L4/5 & II & - \\
7 & $53 / \mathrm{M}$ & 24 & PS & L3/4 & II & - \\
8 & $81 / \mathrm{M}$ & 28 & PS & L3/4 & III & + +: Delayed Union \\
9 & $83 / \mathrm{M}$ & 24 & TS & L4/5 & II & - \\
10 & $51 / \mathrm{F}$ & 20 & PS & L4/5 & II & - \\
11 & $61 / \mathrm{M}$ & 24 & PS & L2/3 & III & + +: Recurrence \\
$\mathrm{n}=11$ & $67.2 \pm 13.4$ & $32.5 \pm 10.1$ & & & & $4(36.4 \%)$ \\
\hline
\end{tabular}

TS: tuberculous spondylodiscitis; PS: pyogenic spondylodiscitis

Table 3. Clinical Details of the Conventional Anterior Open Approach Group (Group C).

\begin{tabular}{ccccccc}
\hline $\begin{array}{c}\text { Case } \\
\text { No. }\end{array}$ & Age (years)/Sex & $\begin{array}{c}\text { Follow-up } \\
\text { (months) }\end{array}$ & Diagnosis & Level & Grade & $\begin{array}{c}\text { Additional Posterior } \\
\text { Instrumentation }\end{array}$ \\
\hline 1 & $50 / \mathrm{M}$ & 34 & TS & L3/4 & III & - \\
2 & $76 / \mathrm{M}$ & 45 & PS & L4/5 & II & - \\
3 & $63 / \mathrm{M}$ & 41 & TS & L4/5 & III & +: Delayed Union \\
4 & $48 / \mathrm{F}$ & 12 & PS & L3/4/5 & III & - \\
5 & $82 / \mathrm{F}$ & 60 & PS & L4/5 & II & - \\
6 & $34 / \mathrm{F}$ & 39 & TS & L2/3/4 & III & - \\
7 & $54 / \mathrm{M}$ & 23 & TS & L4/5 & II & - \\
8 & $79 / \mathrm{F}$ & 50 & TS & L3/4 & III & - \\
9 & $52 / \mathrm{M}$ & 12 & TS & L4/5 & II & - \\
10 & $69 / \mathrm{M}$ & 72 & PS & L2/3 & II & - \\
11 & $71 / \mathrm{M}$ & 12 & TS & L4/5 & II & - \\
12 & $22 / \mathrm{F}$ & 38 & TS & L4/5 & III & - \\
13 & $72 / \mathrm{F}$ & 59 & PS & L3/4/5 & II & + +: Pseudarthrosis \\
n=13 & $60.5 \pm 17.8$ & $38.2 \pm 19.5$ & & & & 2 (15.4\%) \\
\hline
\end{tabular}

TS: tuberculous spondylodiscitis; PS: pyogenic spondylodiscitis

cision was made over the $12^{\text {th }}$ rib from the lateral border of the quadratus lumborum to the lateral border of the rectus abdominus muscle; this incision was varied depending on which portion of the lumbar spine was to be approached. Subcutaneous tissue, fascia, and muscle of the external oblique, internal oblique, transverse abdominus, and transversalis fascia in line with the skin incision were divided, and the retroperitoneal space was accessed. The psoas muscle in the retroperitoneal space was identified and retracted posteriorly to the level of the transverse process, whereas retroperitoneal fat and the ureter were retracted anteriorly to expose the involved vertebra.

In all cases, an autologous iliac crest bone graft was harvested according to the size of the bone defect. In two-level fusion cases, patients underwent a single corpectomy and discectomies craniocaudally. After debridement and sequestrectomy, the bone graft was inserted orthogonally into the defect in a press-fit manner.

\section{Results}

The study cohort consisted of 15 males and 9 females. In Group $\mathrm{M}$, the mean age and duration of follow-up were 67.2 (range 51-84) years and 32.5 (range 20-48) months, respectively. Patient diagnoses and grades of severity were as follows: TS in 3 patients, PS in 8, grade II in 9, and grade III in 2, respectively. Ten patients underwent single-level fusion, and only 1 patient underwent two-level fusion (Table 2). In Group $\mathrm{C}$, the mean age and duration of follow-up were 60.5 (range 34-82) years and 38.2 (range 12-72) months, respectively. Patient diagnoses and grades of severity were as follows: TS in 8 , PS in 5 , grade II in 7 , and grade III in 6, respectively. Ten patients underwent singlelevel fusion, and 3 patients underwent two-level fusion (Table 3).

As shown in Table 4, the mean OT was lower in Group M (162.9 min) than in Group C (174.6 min). EBL was sig- 
Table 4. Summary of Peri- and Postoperative Evaluations.

\begin{tabular}{lccr}
\hline & Group M (n=11) & Group C (n=13) & \multicolumn{1}{c}{$P$} \\
\hline Operative Time (min) & $162.9 \pm 34.1$ & $174.6 \pm 48.0$ & 0.23 \\
Estimated Blood Loss (mL) & $202.1 \pm 161.4$ & $648.3 \pm 569.8$ & $<\mathbf{0 . 0 5}$ \\
Creatine Kinase at 1 POD (IU/L) & $390.9 \pm 203.7$ & $925.5 \pm 585.3$ & $<\mathbf{0 . 0 5}$ \\
Visual Analog Scale (VAS) & & & \\
VAS at 1 POW (mm) & $18.0 \pm 10.6$ & $24.2 \pm 12.9$ & 0.11 \\
VAS at 2 POW (mm) & $9.5 \pm 10.4$ & $22.3 \pm 11.5$ & $<\mathbf{0 . 0 1}$ \\
Union Rate & $7(63.6)$ & $11(84.6)$ & 0.23 \\
Additional Posterior Instrumentation & $4(36.4)$ & $2(15.4)$ & 0.36 \\
JOA Recovery Rate (\%) & $57.6 \pm 10.9$ & $50.8 \pm 14.8$ & 0.11 \\
\hline
\end{tabular}

Mean \pm standard deviation or $\mathrm{n}(\%)$

POD: postoperative day; POW: postoperative week; JOA: Japanese Orthopedic Association

Bold values indicate statistical significance.

nificantly lower in Group M (202.1 mL) than in Group C (648.3 mL). Serum CK on the $1^{\text {st }}$ operative day was reduced in Group M (390.9 IU/L) than in Group C (925.5 IU/L). VAS at the $14^{\text {th }}$ postoperative day was significantly lower in Group M $(9.5 \mathrm{~mm})$ than in Group C $(22.3 \mathrm{~mm})$. Bone union rates were $63.6 \%$ in Group $\mathrm{M}$ and $84.6 \%$ in Group C. Four cases in Group $\mathrm{M}$ and 2 cases in Group $\mathrm{C}$ required additional posterior instrumentation for reinforcement of pseudarthrosis or recurrence of spondylodiscitis. There was no statistical difference between the groups in JOA recovery rates (Table 4).

\section{Case presentation}

The representative case of Group M (Case No. 7) was a 54-year-old male who had severe low back pain and was referred to our institute for PS at L3-4. CT revealed severe osteolytic changes of the endplates with invasion of the vertebral body; the severity of spondylodiscitis was classified as grade II (Fig. 1A-C). The patient underwent lumbar intervertebral debridement and reconstruction surgery using the minimal retroperitoneal approach followed by an autologous iliac crest strut bone graft without posterior instrumentation. OT was $160 \mathrm{~min}$ with an EBL of $95 \mathrm{~mL}$. Culture of the sequestrum revealed the presence of Pseudomonas aeruginosa sp. Radiographic bony fusion was observed within 12 months without any recurrence of infection (Fig. 1D-F).

\section{Discussion}

In this study, we demonstrated the efficacy of a minimal retroperitoneal approach for anterior spinal fusion with an autologous strut bone graft for infectious spondylodiscitis. This technique has an advantage over the conventional open approach as it reduces blood loss, muscle injury, as evidenced by low CK levels, and VAS postoperatively.

An anterior approach to the lumbar spine offers considerable advantages ${ }^{7}$. First, the direct visualization of and efficient access to the anterior column allow complete discectomy, corpectomy, and debridement. Second, the placement of the intervertebral graft in the anterior column redistributes the load anteriorly to provide greater stability with a larger vascular supply, thereby increasing the potential for fusion. Third, there is reduced iatrogenic trauma to the paraspinal musculature, posterior spinal nerves, and posterior bony elements. However, this technique is considerably invasive and is not suitable for elderly patients with multiple comorbidities. To avoid the complications of anterior open surgery, posterior percutaneous suction aspiration or/and endoscopic surgery are performed instead, resulting in less invasion and good clinical outcomes ${ }^{15,16}$. These procedures can prevent further progression of disease, provided that the damage to the endplate is minor or moderate. However, the effectiveness of posterior approach surgery is limited in cases with spondylodiscitis with vertebral body destruction and large osseous defects ${ }^{10)}$.

Oblique or direct lateral lumbar interbody fusions (LLIFs) are now becoming major techniques, especially for the correction of sagittal and coronal plane spinal degenerative deformities. These techniques apply the minimal approach, the efficacy of which has been demonstrated in various studies $^{8,17,18}$. The minimal approach can access the intervertebral space via between the aorta and the anterior edge of the psoas muscle with minimal risk of muscle and plexus injury. The specialized cylindrical retractor with illumination and X-ray fluoroscopy allows the LLIF procedure to be performed correctly and safely in a less invasive manner. Although rare, however, potentially lethal major vascular complications during anterior approaches are possible. Fantini et al. reported the incidence of injury to major abdominal vessels at $2.9 \%$ in their retrospective analysis of 345 procedures of conventional open anterior lumbar spinal surgery ${ }^{19)}$. For LLIF, compared with the supine position, the vascular structures move a significant distance away from the surgical corridor when the patient is positioned in the lateral decubitus position. Nonetheless, vascular injuries are still possible and are probably among the most significant complications of LLIF. Hijji et al. reviewed the overall incidence of vascular complications associated with LLIF as $0.81 \%$ out of 6819 patients with 11,325 levels fused ${ }^{20)}$. Assina et al. reported a fatal intraoperative injury to the great vessels dur- 

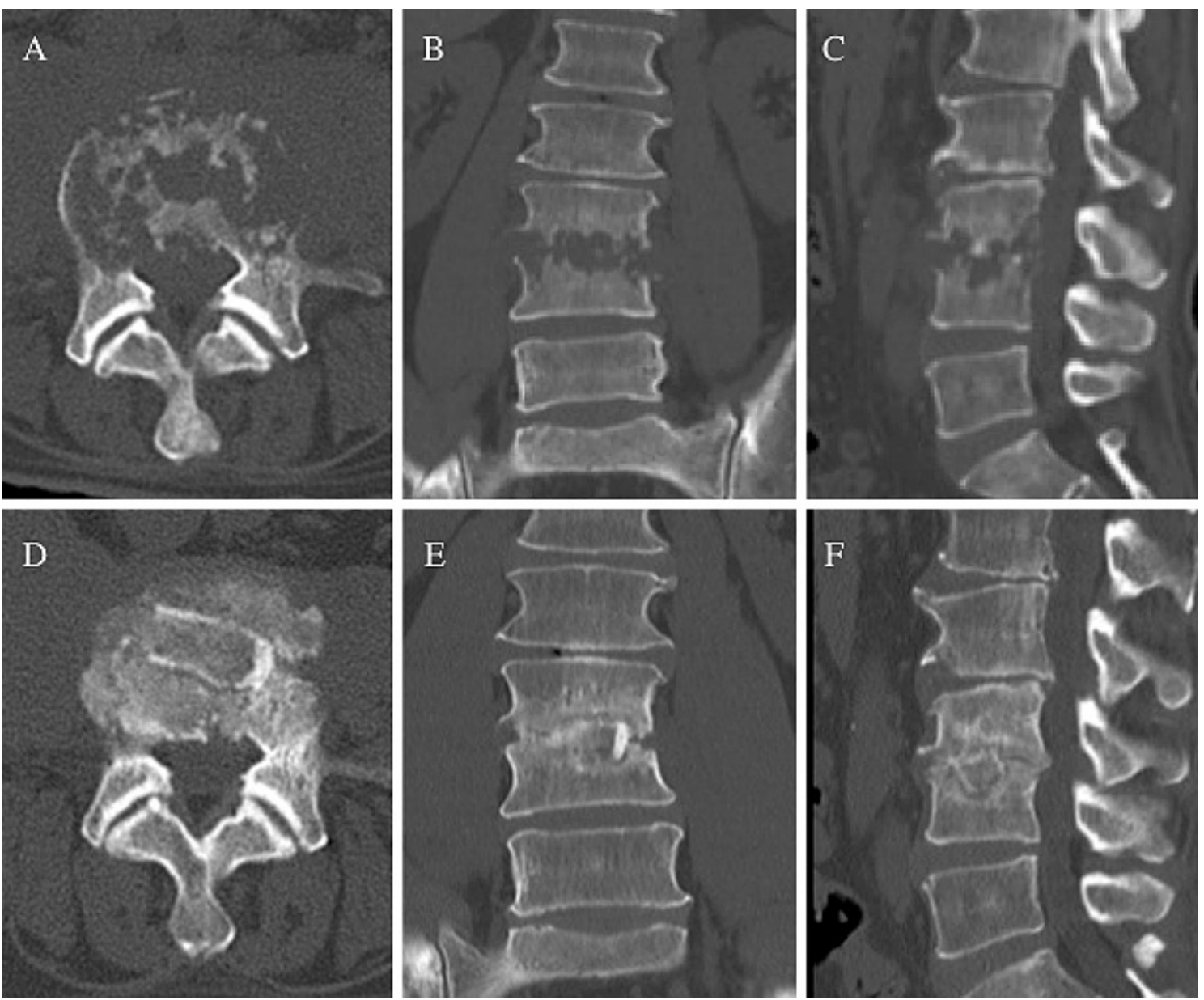

Figure 1. Computed tomography scans showing a representative case of pyogenic spondylodiscitis treated with the minimal retroperitoneal approach (Case No. 7) without posterior instrumentation. Osteolytic changes were seen at the L3-4 intervertebral space (A-C). At 12 months after surgery, a solid intervertebral fusion was obtained (D-F).

ing an LLIF procedure. In the setting of a tubular retractor system with a detachable nonfixed anterior blade, injury to the posterior wall of the vena cava and the right iliac vein confluence was caused $^{21)}$. As this approach gains more popularity and wider usage among experienced and inexperienced surgeons, the real potential for injury to the great vessels must be recognized.

In our series, the clinical outcomes of the minimal approach were equal to those of conventional open surgery. Intraoperative blood loss, $\mathrm{CK}$, which indicates injury to the psoas muscle fibers ${ }^{22}$, and postoperative pain were lower for the minimal approach than for conventional surgery. Several reports demonstrated the superiority of additional posterior instrumentation for reinforcement in single anterior surgery ${ }^{10,23,24}$. Kanayama et al. reported the advantages of anterior spinal reconstruction in cases with osteoporotic vertebral collapse, and $80 \%$ of cases were successfully treated with anterior surgery alone ${ }^{3}$. However, patients with multilevel corpectomies and/or severe osteoporosis require posterior reinforcement. In the present study, $36.4 \%$ of the minimal approach group and $15.4 \%$ of the conventional group received additional posterior instrumentations owing to delayed union or the recurrence of spondylodiscitis. Given our results, we are not able to strongly state a preponderance of the mini- mal approach regarding acquisition of early bony fusion. Thus, in the presence of a larger bone defect, anterior bone grafting alone would not be sufficient to restore and stabilize the spinal column, warranting consideration for additional posterior instrumentation.

The limitations of this study were the small number of patients included. Furthermore, this study was not a casecontrolled study that enabled comparisons between the groups with similar baseline conditions, lacking the power to demonstrate the conclusions properly. Although the statistical power was limited, our findings supported clinical efficacies of this minimal approach to some extent. Another limitation was that patient-reported outcome measures were not assessed, even though almost all patients had a satisfactory clinical outcome. Further study with a randomized and long-term prospective design is warranted to evaluate the efficacy of anterior surgery with the minimal approach.

\section{Conclusion}

Anterior debridement and spinal fusion with an autologous strut bone graft using a minimal retroperitoneal approach is a useful surgical technique that appears to be safe. This technique has the advantages of conventional open sur- 
gery, but reduces blood loss, muscle injury, and VAS postoperatively. However, the surgeon should have experience in performing OLIF and carefully consider vascular injuries and cases with delayed union requiring additional posterior instrumentation.

Conflicts of Interest: The authors declare that there are no relevant conflicts of interest.

Ethical Approval: This study was ethically approved by the Research Ethics Committee of Murayama Medical Center (receipt ID: 12-10). There was no approval code issued by the institutional review board.

Author Contributions: T.K., K.F., and J.Y. collected data, T.K. and A.F.Z. wrote and prepared the manuscript, and S.K., T.A., and Y.Y. supervised the study and manuscript.

Informed Consent: Informed consent was obtained from all participants in this study.

\section{References}

1. Carragee EJ. Pyogenic vertebral osteomyelitis. J Bone Joint Surg Am. 1997;79(6):874-80.

2. Rezai AR, Woo HH, Errico TJ, et al. Contemporary management of spinal osteomyelitis. Neurosurgery. 1999;44(5):1018-25; discussion 25-6.

3. Kanayama M, Ishida T, Hashimoto $T$, et al. Role of major spine surgery using Kaneda anterior instrumentation for osteoporotic vertebral collapse. J Spinal Disord Tech. 2010;23(1):53-6.

4. Batson OV. The function of the vertebral veins and their role in the spread of metastases. Ann Surg. 1940;112(1):138-49.

5. Wiley AM, Trueta J. The vascular anatomy of the spine and its relationship to pyogenic vertebral osteomyelitis. J Bone Joint Surg Br. 1959;41-B:796-809.

6. Sapico FL, Montgomerie JZ. Vertebral osteomyelitis. Infect Dis Clin North Am. 1990;4(3):539-50.

7. Mobbs RJ, Phan K, Thayaparan GK, et al. Anterior lumbar interbody fusion as a salvage technique for pseudarthrosis following posterior lumbar fusion surgery. Global Spine J. 2016;6(1):14-20.

8. Mayer HM. A new microsurgical technique for minimally invasive anterior lumbar interbody fusion. Spine (Phila Pa 1976). 1997;22 (6):691-9; discussion 700.

9. McAfee PC, Regan JJ, Geis WP, et al. Minimally invasive anterior retroperitoneal approach to the lumbar spine. Emphasis on the lateral BAK. Spine (Phila Pa 1976). 1998;23(13):1476-84.

10. Pee YH, Park JD, Choi YG, et al. Anterior debridement and fusion followed by posterior pedicle screw fixation in pyogenic spondylodiscitis: autologous iliac bone strut versus cage. J Neurosurg Spine. 2008;8(5):405-12.
11. Fujibayashi S, Takemoto M, Izeki M, et al. Does the formation of vertebral endplate cysts predict nonunion after lumbar interbody fusion? Spine (Phila Pa 1976). 2012;37(19):E1197-202.

12. Ito $\mathrm{Z}$, Matsuyama $\mathrm{Y}$, Sakai $\mathrm{Y}$, et al. Bone union rate with autologous iliac bone versus local bone graft in posterior lumbar interbody fusion. Spine (Phila Pa 1976). 2010;35(21):E1101-5.

13. Konomi T, Yasuda A, Fujiyoshi $\mathrm{K}$, et al. Incidences and risk factors for postoperative non-union after posterior lumbar interbody fusion with closed-box titanium spacers. Asian Spine J. 2020;14 (1):106-12.

14. Hirabayashi K, Miyakawa J, Satomi K, et al. Operative results and postoperative progression of ossification among patients with ossification of cervical posterior longitudinal ligament. Spine (Phila Pa 1976). 1981;6(4):354-64.

15. Nagata K, Ohashi T, Ariyoshi M, et al. Percutaneous suction aspiration and drainage for pyogenic spondylitis. Spine (Phila Pa 1976). 1998;23(14):1600-6.

16. Ito M, Abumi K, Kotani $\mathrm{Y}$, et al. Clinical outcome of posterolateral endoscopic surgery for pyogenic spondylodiscitis: results of 15 patients with serious comorbid conditions. Spine (Phila Pa 1976). 2007;32(2):200-6.

17. Sato J, Ohtori S, Orita S, et al. Radiographic evaluation of indirect decompression of mini-open anterior retroperitoneal lumbar interbody fusion: oblique lateral interbody fusion for degenerated lumbar spondylolisthesis. Eur Spine J. 2017;26(3):671-8.

18. Orita S, Nakajima T, Konno K, et al. Salvage strategy for failed spinal fusion surgery using lumbar lateral interbody fusion technique: a technical note. Spine Surg Relat Res. 2018;2(1):86-92.

19. Fantini GA, Pappou IP, Girardi FP, et al. Major vascular injury during anterior lumbar spinal surgery: incidence, risk factors, and management. Spine (Phila Pa 1976). 2007;32(24):2751-8.

20. Hijji FY, Narain AS, Bohl DD, et al. Lateral lumbar interbody fusion: a systematic review of complication rates. Spine J. 2017;17 (10):1412-9.

21. Assina R, Majmundar NJ, Herschman Y, et al. First report of major vascular injury due to lateral transpsoas approach leading to fatality. J Neurosurg Spine. 2014;21(5):794-8.

22. Suwa H, Hanakita J, Ohshita N, et al. Postoperative changes in paraspinal muscle thickness after various lumbar back surgery procedures. Neurol Med Chir (Tokyo). 2000;40(3):151-4; discussion 4-5.

23. Hee HT, Majd ME, Holt RT, et al. Better treatment of vertebral osteomyelitis using posterior stabilization and titanium mesh cages. J Spinal Disord Tech. 2002;15(2):149-56; discussion 56.

24. Dimar JR, Carreon LY, Glassman SD, et al. Treatment of pyogenic vertebral osteomyelitis with anterior debridement and fusion followed by delayed posterior spinal fusion. Spine (Phila Pa 1976). 2004;29(3):326-32; discussion 32.

Spine Surgery and Related Research is an Open Access journal distributed under the Creative Commons Attribution-NonCommercial-NoDerivatives 4.0 International License. To view the details of this license, please visit (https://creativeco mmons.org/licenses/by-nc-nd/4.0/). 